\title{
Floristic Diversity Assessment of Major Forest Community of Col. Sher Jung National Park in Himachal Pradesh, India
}

\author{
S. Balaji Naik ${ }^{1 *}$, Vimal Chauhan ${ }^{1}$, Sourav Ranjan Mohapatra ${ }^{2}$ and Kapoor ${ }^{1}$ \\ ${ }^{1}$ Department of Silviculture and Agroforestry, Dr. Y S Parmar University of Horticulture and \\ Forestry, Nauni, Solan-173230, Himachal Pradesh, India \\ ${ }^{2}$ Division of Genetics and Tree Improvement, Forest Research Institute, Dehradun-248006 \\ Uttarakhand, India \\ *Corresponding author
}

\begin{abstract}
A B S T R A C T
The study was carried out at Col. Sher Jung National Park in Sirmour district of Himachal Pradesh, India, during the year 2018-19 with the aim to assess floristic composition, similarity index and dissimilarity index of major forest communities. Eight major forest communities were selected such as Shorea robusta, Eucalyptus tereticornis, Syzygium cumini, Shorea robust + Terminalia tomentosa, Shorea robusta+ Eucalyptus tereticornis, Shorea robusta+ Syzygium cumini, Shorea robusta+ Diospyros melanoxylon and Mixed forest. 90 plant species were recorded in these forests of which 6 were trees, 37 shrubs and 47 forbs. Mixed forest and Shorea robusta+ Syzygium cumini, are similar in shrub composition and have maximum similarity index. Shorea robust + Terminalia tomentosa and Syzygium cumini forest community has minimum similarity index. Shorea robusta + Diospyros melanoxylon and Shorea robusta + Terminalia tomentosa forest has highest similarity in herbs. Whereas, Syzygium cumini and Shorea robusta forest community have lower similarity in herbaceous vegetation.
\end{abstract}

Keywords

Floristic

composition,

Similarity index,

Dissimilarity index, National Park

Article Info

Accepted:

15 May 2020

Available Online:

10 June 2020

\section{Introduction}

Floristic studies have acquired increasing importance in recent years in response to the need of developing countries to assess their plant wealth. Biodiversity maintains balance for planetary and human survival (Virajman et al., 2012). The service provided by biodiversity and ecosystem play a crucial role to sustain our livelihoods and protect our health (Sobuj and Rahman, 2011). Human disturbances through selective logging, wood 
extraction, grazing, fire, and land clearing for permanent agriculture may influence many plant communities and their succession patterns. Long-term changes in patterns and processes in forest systems may lead to losses in their biological diversity and may render them more susceptible to invasion (Hobbs and Huenneke, 1992). Humans have extensively altered the global environment, changing global biogeochemical cycles, transforming land, and enhancing the mobility of biota. Many species have been eliminated from areas dominated by human influences (Chapin FS et al., 2000). The study area although a reserved area is liable to different kind of biotic as well as abiotic interferences which include grazing, human encroachment and other anthropogenic pressures. It becomes very imperative to collect knowledge on the biodiversity and its distribution within the ecologically important and sensitive area for proper conservation and better management of the natural resources. Therefore the study was proposed to study floristic composition, similarity index and dissimilarity index of major forest communities of Col. Sher Jung National Park in Himachal Pradesh.

\section{Materials and Methods}

\section{Study site}

The present study was carried out in the Col. Sher Jung National Park (Fig:1) which encompasses an area of 27.88 sq. $\mathrm{km}$ in the Sirmour district of Himachal Pradesh. It is exactly located between latitudes $30^{\circ}$ $28^{\prime} 13^{\prime}, \mathrm{N}$ to $30^{0} 23^{\prime} 31^{\prime}, \mathrm{N}$ and longitude $77^{0} 28^{\prime} 43^{\prime}$ ' $\mathrm{E}$ to $77^{0} 27^{\prime} 40^{\prime}$ ' $\mathrm{E}$. It lies in Paonta valley of Himachal Pradesh which shares boundary with Kalesar National Park and Rajaji National Park.

National park shows a wide geoenvironmental variation. In location; annual minimum and maximum temperature ranges from $3^{\circ} \mathrm{C}$ - $40^{\circ} \mathrm{C}$, mean annual rainfall about $1200 \mathrm{~mm}$ and relative humidity varies from 26 per cent in summer to 90 per cent during monsoon. Studied area is having an elevation range of $350 \mathrm{amsl}$. to 700amsl. which is composed of unconsolidated siltstone, sandstone and conglomerate.

\section{Sampling protocol}

The study was carried out at Col. Sher Jung National Park in Sirmour district of Himachal Pradesh, India, during the year 2018-19. The major forest species that represent the Col. Sher Jung National Park are Shorea robusta (sal), Eucalyptus tereticornis (Eucalyptus) Terminalia tomentosa (sain) Syzygium cumini (Jamun) and Diospyros melanoxylon (tendu). The species occur either pure or mixed with other important or associated species.

Based on species dominance, eight forest communities were selected i.e. Shorea robusta forest, Eucalyptus tereticornis, Syzygium cumini forest, Shorea robusta+ Terminalia tomentosa forest, Shorea robusta+ Eucalyptus tereticornis, Shorea robusta+ Syzygium cumini forest, Shorea robusta+ Diospyros melanoxylon and Mixed forest.

Community analysis was carried out during rainy season when majority of the plants were at the peak of their growth. In every forest community type, 3 quadrats of $31.62 \mathrm{~m} \mathrm{X}$ $31.62 \mathrm{~m} \mathrm{(0.1} \mathrm{ha)} \mathrm{size} \mathrm{were} \mathrm{randomly} \mathrm{laid} \mathrm{to}$ study tree species. The tree species includes all the saplings, poles and trees present in the study area. The shrub and herbaceous species were studied by laying 3 quadrats randomly in each forest community type. In each quadrat, a sub-quadrat of $5 \mathrm{~m}$ X $5 \mathrm{~m}$ ( $25 \mathrm{sq} \mathrm{m})$ size for shrubs and a sub-quadrat of $1 \mathrm{~m}$ X $1 \mathrm{~m}$ (1sq m) for herbaceous vegetation were selected. Indices of similarity and dissimilarity were calculated following (Mishra, 1989). 


\section{Results and Discussion}

\section{Floristic composition}

Forest composition, community structure and diversity patterns are important ecological attribues correlated with prevailing environmental as well anthropogenic variables (Ahmad I et al., 2010; Bisht AS and Bhat AB, 2011). The major forest communities that existed in the Col. Sher Jung National Park are Shorea robusta, Eucalyptus tereticornis, Syzygium cumini, Shorea robust + Terminalia tomentosa, Shorea robusta+ Eucalyptus tereticornis, Shorea robusta+ Syzygium cumini, Shorea robusta+ Diospyros melanoxylon and Mixed forest (Table $1 \& 2$ ).

Plant families representation in the flora revealed that in this region tree species are primarily from Dipterocarpaceae, Myrtaceae, Combretaceae, Ebenaceae, Euphorbiaceae, shrubs from Acanthaceae, Asparagaceae, Asteraceae, Apocynaceae, Euphorbiaceae, Fabaceae, Lamiaceae and Rosaceae and Solanaceae whereas, herbaceous flora belonged to Asparagaceae, Asteraceae, Fabaceae, Malvaceae and Phyllanthaceae. Similar dominance of the plant farmilies in forests of western Himalayan region has been reported earlier (Kala and Uniyal, 1999). The data pertaining to floristic composition of is given in Table 2. For different forests communities, Shorea robusta, Shorea robust + Terminalia tomentosa and Mixed forest community shows the maximum (35) herbaceous vegetation. Whereas, in other forests community, 18 to 33 only. It is in line of findings earlier in Shorea robusta forests of West Bengal, (Satya Prakash Singh Kushwaha and Subrata Nandy, 2012) and also in Eastern Himalaya Shorea robusta forests, respectively has been reported by Uma Shankar (2001).

Table.1 The major forest community in Col. Sher Jung National Park

\begin{tabular}{|l|l|l|}
\hline \multicolumn{1}{|c|}{ Scientific name } & Common name & Family \\
\hline Shorea robusta & Sal & Dipterocarpaceae \\
\hline Eucalyptus tereticornis & Eucalyptus & Myrtaceae \\
\hline Syzygium cumini & Jamun & Myrtaceae \\
\hline Terminalia tomentosa & Sain & Combretaceae \\
\hline Diospyros melanoxylon & Tendu & Ebenaceae \\
\hline
\end{tabular}

Table.2 Floristic composition of major forest community type in Col. Sher Jung National Park

\begin{tabular}{|c|l|l|}
\hline Sr. No & Scientific Name & Family \\
\cline { 2 - 3 } & Trees & \\
\hline $\mathbf{1}$ & Diospyros melanoxylon & Ebenaceae \\
\hline $\mathbf{2}$ & Eucalyptus tereticornis & Myrtaceae \\
\hline $\mathbf{3}$ & Mallotus philippinensis & Euphorbiaceae \\
\hline $\mathbf{4}$ & Shorea robusta & Dipterocarpaceae \\
\hline $\mathbf{5}$ & Syzyium cumini & Myrtaceae \\
\hline $\mathbf{6}$ & Terminalia tomentosa & Combretaceae \\
\hline
\end{tabular}




\begin{tabular}{|c|c|c|}
\hline Sr. No & Scientific Name & Family \\
\hline & Shrubs & \\
\hline 1 & Adhatoda vasica & Acanthaceae \\
\hline 2 & Ardisia solanacea & Primulaceae \\
\hline 3 & Agave cantula & Asparagaceae \\
\hline 4 & Asparagus adsecndes & Asparagaceae \\
\hline 5 & Baliospermum montanum & Euphorbiaceae \\
\hline 6 & Barleria strigosa & Acanthaceae \\
\hline 7 & Boehmeria frutescens & Urticaceae \\
\hline 8 & Calamus tenius & Arecaceae \\
\hline 9 & Calotropis procera & Apocynaceae \\
\hline 10 & Cassia occidentalis & Fabaceae \\
\hline 11 & Carissa opaca & Apocynaceae \\
\hline 12 & Callicarpa macrophylla & Lamiaceae \\
\hline 13 & Clerodendrum viscosum & Lamiaceae \\
\hline 14 & Coffea benghalensis & Rubiaceae \\
\hline 15 & Colebrookia oppostifolia & Lamiaceae \\
\hline 16 & Dendrolobium triangulare & Fabaceae \\
\hline 17 & Desmodium gangeticum & Fabaceae \\
\hline 18 & Desmodium pulchellum & Fabaceae \\
\hline 19 & Eupatorium adenophorum & Asteraceae \\
\hline 20 & Flemingia bracteata & Fabaceae \\
\hline 21 & Flemingia chappar & Fabaceae \\
\hline 22 & Holarrhena antidysentrica & Apocynaceae \\
\hline 23 & Indigofera gerardiana & Fabaceae \\
\hline 24 & Iротеа atropurpurea & Convolvulaceae \\
\hline 25 & Ipomea carnea & Convolvulaceae \\
\hline 26 & Lantana Camara & Verbenaceae \\
\hline 27 & Murraya koenigii & Rutaceae \\
\hline 28 & Phlogacanthus thyrsiflorus & Acanthaceae \\
\hline 29 & Randia uliginosa & Rubiaceae \\
\hline 30 & Rubus ellipticus & Rosaceae \\
\hline 31 & Solanum hispidum & Solanaceae \\
\hline 32 & Solanum torvum & Solanaceae \\
\hline 33 & Toddalia asiatica & Rutaceae \\
\hline 34 & Urena labata & Malvaceae \\
\hline 35 & Vitex negundo & Lamiaceae \\
\hline 36 & Woodfordia floribunda & Lythraceae \\
\hline 37 & Xanthium strumarium & Asteraceae \\
\hline
\end{tabular}




\begin{tabular}{|c|c|c|}
\hline Sr. No & Scientific Name & Family \\
\hline & Herbs/Grasses/Climbers & \\
\hline 1 & Achyranthes aspera & Amaranthaceae \\
\hline 2 & Acrocephalus Capitatus & Lamiaceae \\
\hline 3 & Alternanthera sessilis & Amaranthaceae \\
\hline 4 & Asparagus racemosus wild & Asparagaceae \\
\hline 5 & Ageratum conyzoides & Asteraceae \\
\hline 6 & Bidnes biternata & Asteraceae \\
\hline 7 & Borreria articularis & Rubiaceae \\
\hline 8 & Commelina benghalensis & Commelinaceae \\
\hline 9 & Cynaglossum lanceolatum & Boraginaceae \\
\hline 10 & Corchorus aestuans & Malvaceae \\
\hline 11 & Desmodium heterocarpan & Fabaceae \\
\hline 12 & Desmodium triliflorum & Fabaceae \\
\hline 13 & Emilia sanchifolia & Asteraceae \\
\hline 14 & Euphorbia hirta & Euphorbiaceae \\
\hline 15 & Malvastrum coromandelianum & Malvaceae \\
\hline 16 & Mazus ragosus & Mazaceae \\
\hline 17 & Mimosa pudica & Fabaceae \\
\hline 18 & Nepeta hindostana & Lamiaceae \\
\hline 19 & Oxalis corniculata & Oxalidaceae \\
\hline 20 & Peperomia pellucid & Piperaceae \\
\hline 21 & Phyllantus virgatus & Phyllanthaceae \\
\hline 22 & Phyllanthus urinaria & Phyllanthaceae \\
\hline 23 & Portulaca pilosa & Portulacaceae \\
\hline 24 & Sida cordata & Malvaceae \\
\hline 25 & Sida cordifolia & Malvaceae \\
\hline 26 & Sida rhomboidea & Malvaceae \\
\hline 27 & Spilanthes Paniculata & Asteraceae \\
\hline 28 & Syndrella vialis & Asteraceae \\
\hline 29 & Tridax procumbens & Asteraceae \\
\hline 30 & Triumfetta rhomboidea & Malvaceae \\
\hline 31 & Vernonia cinerea & Asteraceae \\
\hline 32 & Xanthium indicum & Asteraceae \\
\hline 33 & Cynodon dactylon & Poaceae \\
\hline 34 & Cyprus kyllingia & Cyperaceae \\
\hline 35 & Digitaria cilaris & Poaceae \\
\hline 36 & Ergrostis minor & Poaceae \\
\hline 37 & Eulaliopsis binata & Poaceae \\
\hline 38 & Oplismenus composites & Poaceae \\
\hline 39 & Paspalidium flavidum & Poaceae \\
\hline 40 & Saccharum spontaneum & Poaceae \\
\hline 41 & Cyperus distans & Cyperaceae \\
\hline
\end{tabular}




\begin{tabular}{|l|l|l|}
\hline $\mathbf{4 2}$ & Cyperus rotundus & Cyperaceae \\
\hline $\mathbf{4 3}$ & Cryptolepis buchananii & Ascepiadaceae \\
\hline $\mathbf{4 4}$ & Milletia auriculata & Fabaceae \\
\hline $\mathbf{4 5}$ & Pueraria tuberose & Fabaceae \\
\hline $\mathbf{4 6}$ & Adiatum venustum & Pteridaceae \\
\hline $\mathbf{4 7}$ & Chelilanthes farinose & Pteridaceae \\
\hline
\end{tabular}

Table.3 Shrubs species under different forest community in Col. Sher Jung National Park (H.P)

\begin{tabular}{|c|c|c|c|c|c|c|c|c|c|}
\hline $\begin{array}{l}\text { Sr. } \\
\text { No. }\end{array}$ & Shrub & Sal & $\begin{array}{c}\text { Eucalypt } \\
\text { us }\end{array}$ & $\begin{array}{c}\text { Jamu } \\
\mathbf{n}\end{array}$ & $\begin{array}{l}\text { Sal + } \\
\text { Sain }\end{array}$ & $\begin{array}{c}\text { Sal+ } \\
\text { Eucalyptus }\end{array}$ & $\begin{array}{c}\text { Sal + } \\
\text { Jamun }\end{array}$ & $\begin{array}{c}\text { Sal + } \\
\text { Tendu }\end{array}$ & $\begin{array}{c}\text { Mixe } \\
\text { d } \\
\text { Fores } \\
\mathbf{t}\end{array}$ \\
\hline 1 & Adhatoda vasica & + & - & - & + & + & + & + & + \\
\hline 2 & Ardisia solanacea & + & - & + & + & - & + & + & + \\
\hline 3 & Agave cantala & - & + & + & - & + & + & + & + \\
\hline 4 & Asparagus adsecndes & + & - & + & - & - & + & + & + \\
\hline 5 & Baliospermum montanum & + & - & - & + & - & - & - & + \\
\hline 6 & Barleria strigosa & - & - & - & + & - & - & - & - \\
\hline 7 & Boehmeria frutescens & - & - & - & + & - & + & - & + \\
\hline 8 & Calamus tenius & - & + & + & - & + & - & + & + \\
\hline 9 & Calotropis procera & - & + & + & - & + & - & - & - \\
\hline 10 & Cassia occidentalis & - & - & - & + & - & - & - & + \\
\hline 11 & Carissa opaca & + & + & + & - & - & + & - & + \\
\hline 12 & Callicarpa macrophylla & - & - & + & - & - & - & + & - \\
\hline 13 & Clerodendrum viscosum & + & - & - & - & + & - & - & - \\
\hline 14 & Coffea benghalensis & - & - & - & - & - & + & + & + \\
\hline 15 & Colebrookea oppostifolia & - & - & - & - & - & - & + & - \\
\hline 16 & Dendrolobium triangulare & - & - & + & - & - & + & - & - \\
\hline 17 & Desmodium gangeticum & - & - & + & - & - & - & - & - \\
\hline 18 & Desmodium pulchellum & - & - & + & - & - & + & - & - \\
\hline 19 & Eupatorium adenophorum & + & - & - & - & - & - & + & + \\
\hline 20 & Flemingia bracteata & - & - & - & - & + & - & - & - \\
\hline 21 & Flemingia chappar & - & + & + & - & - & + & - & - \\
\hline 22 & Holarrhena antidysenterica & + & - & - & + & - & - & + & + \\
\hline 23 & Indigofera gerardiana & - & + & + & + & - & - & + & - \\
\hline 24 & Ipomea atropurpurea & - & - & - & - & - & - & + & + \\
\hline 25 & Ipomea carnea & - & - & - & + & - & + & - & + \\
\hline 26 & Lantana Camara & + & + & - & + & + & - & - & - \\
\hline 27 & Murraya koenigii & + & + & + & + & + & + & + & + \\
\hline 28 & Phlogacanthus thyrsiflorus & + & - & - & - & - & - & + & - \\
\hline 29 & Randia uliginosa & - & - & - & + & - & - & - & - \\
\hline 30 & Rubus ellipticus & + & + & - & - & + & - & - & + \\
\hline 31 & Solanum hispidum & + & - & - & - & + & + & - & + \\
\hline 32 & Solanum torvum & + & + & - & + & + & + & + & + \\
\hline 33 & Toddalia asiatica & - & - & + & - & - & - & - & - \\
\hline 34 & Urena lobata & + & - & - & + & - & - & + & - \\
\hline 35 & Vitex negundo & - & - & + & - & - & - & + & - \\
\hline 36 & Woodfordia floribunda & - & - & - & + & - & - & + & - \\
\hline \multirow[t]{2}{*}{37} & Xanthium strumarium & - & - & - & + & - & - & + & - \\
\hline & Total & 15 & 10 & 15 & 16 & 11 & 14 & 18 & 18 \\
\hline
\end{tabular}


Table.4 Herbaceous vegetation under major forest community types in Col. Sher Jung National Park

\begin{tabular}{|c|c|c|c|c|c|c|c|c|c|}
\hline $\begin{array}{l}\text { Sr. } \\
\text { No. }\end{array}$ & Herbs/ Grasses/Climbers & Sal & $\begin{array}{l}\text { Eucal } \\
\text { yptus }\end{array}$ & $\begin{array}{l}\text { Ja } \\
\text { mun }\end{array}$ & $\begin{array}{l}\text { Sal+ } \\
\text { Sain }\end{array}$ & $\begin{array}{l}\text { Sal+ } \\
\text { Eucal } \\
\text { yptus }\end{array}$ & $\begin{array}{l}\text { Sal+ } \\
\text { Jamun }\end{array}$ & $\begin{array}{l}\text { Sal+ } \\
\text { Tend } \\
\text { u }\end{array}$ & $\begin{array}{l}\text { Mixed } \\
\text { Forest }\end{array}$ \\
\hline 1 & Achyranthes aspera & + & + & + & + & + & + & + & + \\
\hline 2 & Acrocephalus Capitatus & + & - & - & + & - & - & + & + \\
\hline 3 & Alternanthera sessilis & + & + & + & + & + & + & + & + \\
\hline 4 & Asparagus racemosus wild & - & - & + & - & - & + & - & - \\
\hline 5 & Ageratum conyzoides & + & + & - & + & + & - & + & + \\
\hline 6 & Bidens biternata & - & - & + & + & - & + & + & + \\
\hline 7 & Borreria articularis & + & + & + & + & + & + & + & + \\
\hline 8 & Commelina benghalensis & - & - & - & + & - & - & + & + \\
\hline 9 & Cynoglossum lanceolatum & - & - & - & - & - & - & - & + \\
\hline 10 & Corchorus aestuans & + & + & - & + & + & + & + & - \\
\hline 11 & Desmodium heterocarpon & + & + & + & + & + & + & + & + \\
\hline 12 & Desmodium triflorum & + & - & - & + & + & + & + & + \\
\hline 13 & Emilia sonchifolia & + & - & - & + & + & + & + & + \\
\hline 14 & Euphorbia hirta & - & - & + & - & - & + & - & - \\
\hline 15 & Malvastrum coromandelianum & + & + & - & - & + & - & + & - \\
\hline 16 & Mazus rugosus & - & - & - & + & - & - & - & + \\
\hline 17 & Mimosa pudica & + & + & - & - & + & + & + & + \\
\hline 18 & Nepeta hindostana & - & - & - & + & - & - & + & + \\
\hline 19 & Oxalis corniculata & + & - & - & - & - & + & + & + \\
\hline 20 & Peperomia pellucidaaa & + & - & - & + & - & - & + & + \\
\hline 21 & Phyllanthus virgatus & + & + & - & + & + & - & + & + \\
\hline 22 & Phyllanthus urinaria & - & + & + & - & + & + & - & - \\
\hline 23 & Portulaca pilosa & - & - & + & - & - & - & - & - \\
\hline 24 & Sida cordata & + & - & - & + & + & - & - & + \\
\hline 25 & Sida cordifolia & + & - & + & + & - & - & - & - \\
\hline 26 & Sida rhomboidea & + & + & - & + & + & + & + & + \\
\hline 27 & Spilanthes Paniculata & - & - & + & - & - & + & - & - \\
\hline 28 & Syndrella vialis & + & + & + & + & + & + & + & + \\
\hline 29 & Tridax procumbens & + & + & - & + & + & - & + & + \\
\hline 30 & Triumfetta rhomboidea & + & - & + & + & - & + & + & + \\
\hline 31 & Vernonia cinerea & + & + & + & + & + & + & + & + \\
\hline 32 & Xanthium indicum & - & - & + & - & - & - & - & - \\
\hline 33 & Cynodon dactylon & + & + & + & + & + & + & + & + \\
\hline 34 & Cyprus kyllingia & + & + & + & + & + & + & + & + \\
\hline 35 & Digitaria ciliaris & + & - & - & + & - & - & + & + \\
\hline 36 & Eragrostis minor & + & - & + & + & - & + & - & + \\
\hline 37 & Eulaliopsis binata & + & - & - & - & - & - & - & - \\
\hline 38 & Oplismenus composites & + & + & - & + & - & - & + & + \\
\hline 39 & Paspalidium flavidum & - & - & + & - & - & + & - & - \\
\hline 40 & Saccharum spontaneum & - & - & + & - & - & - & - & - \\
\hline 41 & Cyperus distans & + & + & - & + & + & - & - & + \\
\hline 42 & Cyperus rotundus & + & - & + & - & - & + & + & + \\
\hline 43 & Cryptolepis buchananii & + & - & - & + & - & - & + & + \\
\hline 44 & Millettia auriculata & + & - & - & - & - & - & + & + \\
\hline 45 & Pueraria tuberose & + & - & - & - & - & - & - & - \\
\hline 46 & Adiatum venustum & + & - & + & + & - & + & + & + \\
\hline \multirow[t]{2}{*}{47} & Cheilanthes farinose & + & - & - & + & - & - & + & + \\
\hline & Total & 35 & 18 & 22 & 35 & 21 & 25 & 33 & 35 \\
\hline
\end{tabular}


Table.5 Index of Similarity and Dissimilarity of Shrubs in Different Forest Communities

\begin{tabular}{|c|c|c|c|c|c|c|c|c|}
\hline & \multicolumn{8}{|c|}{ Index of similarity \& dissimilarity of shrubs in different forest community type } \\
\hline D & Sal & Eucalyptus & Jamun & Sal + Sain & $\begin{array}{c}\text { Sal + } \\
\text { Eucalyptus }\end{array}$ & $\begin{array}{c}\text { Sal + } \\
\text { Jamun }\end{array}$ & $\begin{array}{c}\text { Sal + } \\
\text { Tendu }\end{array}$ & Mixed \\
\hline Sal & - & 0.24 & 0.26 & 0.51 & 0.53 & 0.48 & 0.48 & 0.66 \\
\hline Eucalyptus & 0.76 & - & 0.48 & 0.23 & 0.57 & 0.41 & 0.35 & 0.42 \\
\hline Jamun & 0.74 & 0.52 & - & 0.19 & 0.3 & 0.55 & 0.48 & 0.36 \\
\hline Sal + Sain & 0.49 & 0.77 & 0.81 & - & 0.29 & 0.4 & 0.47 & 0.41 \\
\hline $\begin{array}{l}\text { Sal + } \\
\text { Eucalyptus }\end{array}$ & 0.47 & 0.43 & 0.7 & 0.71 & - & 0.4 & 0.34 & 0.48 \\
\hline $\begin{array}{l}\text { Sal + } \\
\text { Jamun }\end{array}$ & 0.52 & 0.59 & 0.45 & 0.6 & 0.6 & - & 0.37 & 0.68 \\
\hline $\begin{array}{l}\text { Sal + } \\
\text { Tendu }\end{array}$ & 0.52 & 0.65 & 0.52 & 0.53 & 0.66 & 0.63 & - & 0.55 \\
\hline Mixed & 0.34 & 0.58 & 0.64 & 0.59 & 0.52 & 0.32 & 0.45 & - \\
\hline
\end{tabular}

Table.6 Index of Similarity and Dissimilarity of Herbs in Different Forest Communities

\begin{tabular}{|l|c|c|c|c|c|c|c|c|}
\hline \multirow{2}{*}{ D } & \multicolumn{6}{|c|}{ Index of similarity \& dissimilarity of shrubs in different forest community type } \\
\cline { 2 - 10 } & Sal & Eucalyptus & Jamun & Sal + Sain & $\begin{array}{c}\text { Sal + } \\
\text { Eucalyptus }\end{array}$ & $\begin{array}{c}\text { Sal + } \\
\text { Jamun }\end{array}$ & $\begin{array}{c}\text { Sal + } \\
\text { Tendu }\end{array}$ & Mixed \\
\hline Sal & - & 0.71 & 0.45 & 0.88 & 0.71 & 0.63 & 0.88 & 0.82 \\
\hline Eucalyptus & 0.29 & - & 0.5 & 0.6 & 0.92 & 0.6 & 0.62 & 0.56 \\
\hline Jamun & 0.55 & 0.5 & - & 0.49 & 0.46 & 0.8 & 0.5 & 0.49 \\
\hline Sal + Sain & 0.12 & 0.4 & 0.51 & - & 0.64 & 0.66 & 0.95 & 0.94 \\
\hline $\begin{array}{l}\text { Sal + } \\
\text { Eucalyptus }\end{array}$ & 0.29 & 0.08 & 0.54 & 0.36 & - & 0.65 & 0.66 & 0.64 \\
\hline $\begin{array}{l}\text { Sal + } \\
\text { Jamun }\end{array}$ & 0.37 & 0.4 & 0.2 & 0.34 & 0.35 & - & 0.68 & 0.63 \\
\hline Sal + Tendu & 0.12 & 0.38 & 0.5 & 0.05 & 0.34 & 0.32 & - & 0.88 \\
\hline Mixed & 0.18 & 0.44 & 0.51 & 0.06 & 0.36 & 0.37 & 0.12 & - \\
\hline
\end{tabular}

Fig.1 Detail map of Col. Sher Jung National Park, Simbalbara, Sirmour, H.P., India (Inset: Location map of Sirmour in H.P.)

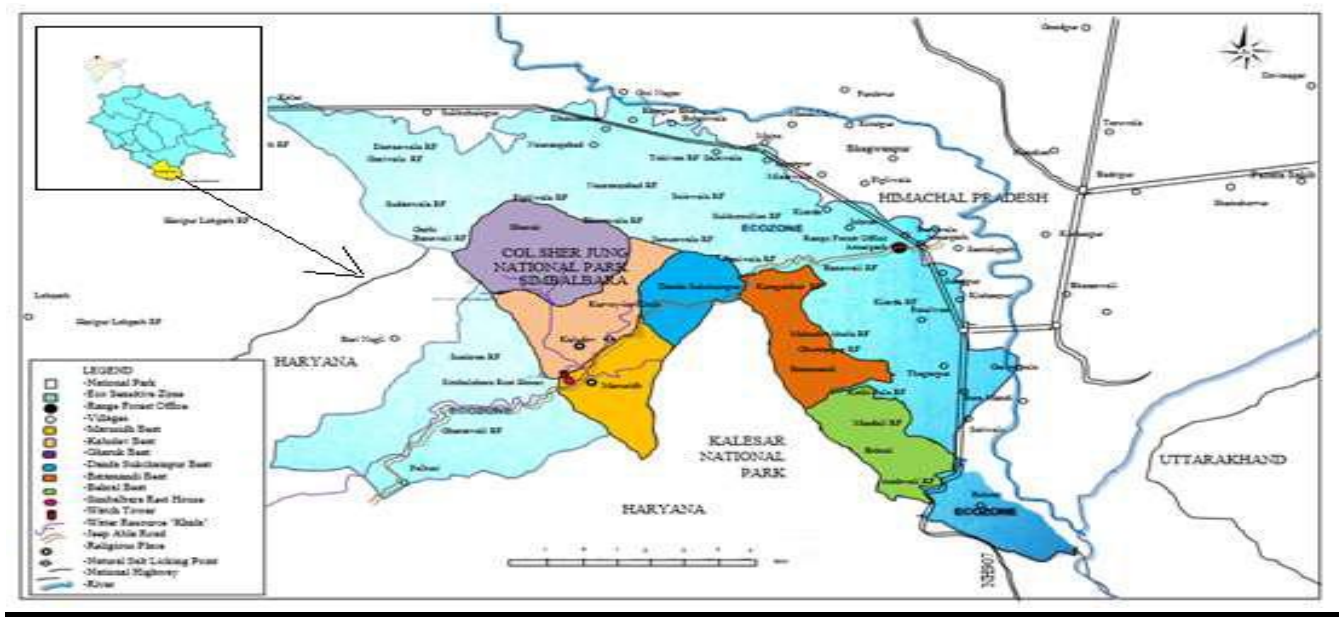




\section{Similarity and dissimilarity index}

The shrubs that existed under major forest communities are given in Table 3. Maximum shrubs were recorded in Shorea robusta+ Diospyros melanoxylon and Mixed forest community (18) followed by Shorea robust + Terminalia tomentosa forest community (16), Shorea robust and Syzygium cumini forest community (15), Shorea robusta+ Syzygium cumini forest community (14), Shorea robusta+ Eucalyptus tereticornis (11), Eucalyptus tereticornis community (10) in descending order.

Herbaceous vegetation that existed in eight major forest communities of Col. Sher Jung National Park is given in Table 4. Maximum herbaceous vegetation was recorded in Shorea robust, Shorea robust + Terminalia tomentosa, and Mixed forest community (35) followed by Shorea robusta+ Diospyros melanoxylon forest community (33), Shorea robusta+ Syzygium cumini forest community (25), Syzygium cumini forest community (22), Shorea robusta+ Eucalyptus tereticornis forest community (21) and Eucalyptus tereticornis community type (18) in descending order. Different forests had varied herbaceous composition but still some species were common to them like, Achyranthes aspera, Alternanthera sessilis, Ageratum conyzoides, Borreria articularis and Desmodium heterocarpon. The presence of any species in any area is determined by the prevailing environmental conditions and its tolerance and adaptation (Bhandari BS et al., 1999).

The value of similarity index (Table 5) for shrubs (0.68) was observed maximum between Mixed forest and Shorea robusta+ Syzygium cumini forest and minimum (0.19) in Shorea robusta + Terminalia tomentosa and Syzygium cumini forest community.
While in case of herbs, maximum similarity (0.95) in Shorea robusta+ Diospyros melanoxylon forest and Shorea robusta + Terminalia tomentosa forest and minimum (0.45) between Syzygium cumini forest and Shorea robusta forest (Table 6). This similarity between different forest community types may be due to same altitudinal zone and similar type of habitat conditions. Less difference in the value of similarity index indicated that growth forms in the stands responded in a similar fashion (Adhikari BS et al., 1991). Whereas, minimum similarity observed between different sites may be due to different climatic conditions and different type of habitat (Itow S and Nakanishi K, 1980) reported that large area contained more varied habitat types then smaller ones, and each of the habitat type supports a specific set of its own, which is more or less different from that of other habitat types. (Suyal S et al., 2010) opined that close proximity results in high similarity index of vegetation. The same could be related to the grouping of forests based on similarity index in the present study.

In conclusion, there were 6 species of tree, 37 species of shrub and 47 species of herb belonging to 42 families in the Col. Sher Jung National Park. Mixed forest community is denser as compared to Syzygium cumini, Shorea robusta+ Syzygium cumini, Shorea robusta+ Diospyros melanoxylon, Shorea robusta + Terminalia tomentosa, Shorea robusta+ Eucalyptus tereticornis, Shorea robusta and Eucalyptus tereticornis community in descending order. Shrub density is higher in Shorea robusta forest community as compared to Shorea robusta + Terminalia tomentosa, Shorea robusta+ Diospyros melanoxylon, Shorea robusta+ Syzygium cumini, Mixed, Syzygium cumini, Shorea robusta + Eucalyptus tereticornis and Eucalyptus tereticornis forest community in descending order. Herb density is higher in 
Shorea robusta+ Diospyros melanoxylon forest community compared to Shorea robusta + Terminalia tomentosa, Shorea robusta, Mixed, Shorea robusta+ Syzygium cumini, Syzygium cumini, Shorea robusta+ Eucalyptus tereticornis and Eucalyptus tereticornis forest community. Mixed forest and Shorea robusta+ Syzygium cumini forest community have maximum shrub similarity index. Syzygium cumini forest and Shorea robusta forest community has minimum similarity index. Shorea robusta+ Diospyros melanoxylon forest and Shorea robusta + Terminalia tomentosa forest has highest similarity in herbs. Whereas, Syzygium cumini forest and Shorea robusta forest community have lower similarity in herbaceous vegetation.

\section{References}

Adhikari BS, Rikhari HC, Rawat YS, Singh SP (1991) High altitude forest: composition, diversity and profile structure in a part of Kaumaun Himalaya, Trop Ecol 32: 8697.

Ahmad I, Ahmad MSA, Husain M, Ashraf M, Ashraf MY, Hameed M (2010) Spatiotemporal aspects of plant community structure in open scrub land of sub mountatious Himalaya Plenteous. Pak Jour Bot 42: 3431-3440.

Bhandari BS, Nautiyal DC, Gaur RD (1999) Structural attributes and productivity potential of an alpine pasture of Garhwal Himalaya. J Ind Soc $78: 321-329$.

Bisht AS, Bhat AB (2011) Effect of human activities, and environmental changes in an alpine vegetation of district Chamoli, Garhwal Himalaya, Uttarakhand, India. World Rural Observ 3: 64-71.

Chapin FS, Zavaleta ES, Eviner VT, Naylor RL, Vitousek PM, Reynolds HL (2000) Consequences of changing biodiversity. Nature 405: 234-242.
Hobbs RJ, Huenneke LF (1992) Disturbances, diversity and invasion: implication for conservation. Conserv Biol 6: 324-337.

Itow S, Nakanishi K (1980) Floristic and vegetation diversity of epilithic bryophyte communities insular biography. Jap J Ecol 30: 45-54.

Kala CP and Uniyal VK. 1999. Forest vegetation along an altitudinal gradient in the valley of Flower National Park and its vicinity of Western Himalayan. Annals of Forestry 7: 60-69.

Mishra KC (1989) Mannual of plant ecology. 3rd (edn) Oxford \& IBH Publ Co Pvt Ltd, New Delhi, pp 193. Gairola S, Rawat RS, Todaria NP (2008) Forest vegetation patterns along altitudinal gradient in sub alpine zone of west Himalaya, India. Afr J Pl Sci 2: 42-48.

Satya Prakash Singh Khushwaha and Subrata Nandy. 2012. Species diversity and community structure in sal (Shorea robusta) forests of two different rainfall regimes in West Bengal, India. Article in Biodiversity Conservation.

Sobuj NA, Rahman M (2011) Assessment of plant diversity in khadimnagar national park of Bangladesh. Int J Environm Sci 2: 79-91.

Suyal S, Sharma CM, Gairola S, Ghildiyal SK, Rana CS, Butola DS (2010) Phytodiversity (Angiosperms and gymnosperms) in Chaurangikhal forest of Garhwal Himalaya, Uttarakhand, India. Ind J Sci and Technol $3: 267-275$.

Uma Shankar. 2001. A case of high tree diversity in sal (Shorea robusta)dominated lowland forest of Eastern Himalaya: Floristic composition, regeneration and conservation. Article in Current Science.

Virajman, Verma RK, Chauhan NS, Kapoor KS (2012) Phytosociological attributes of porang valley in lippaasrang wildlife sanctuary of district Kinnaur, Himachal Pradesh. Ann For 20: 1-16. 


\section{How to cite this article:}

Balaji Naik, S., Vimal Chauhan, Sourav Ranjan Mohapatra and Kapoor. 2020. Floristic Diversity Assessment of Major Forest Community of Col. Sher Jung National Park in Himachal Pradesh, India. Int.J.Curr.Microbiol.App.Sci. 9(06): 682-692. doi: https://doi.org/10.20546/ijcmas.2020.906.087 